4 Grandas Perez FJ, Jenner PG, Nomoto $M$, et al. (+)-4-Propyl-9-Hydroxynaphthoxazine in Parkinson's Disease. Lancet 1986;i: 906.

5 Temlett JA, Quinn QN, Marsden CD, Lataste $\mathrm{X}$, Jaton AL. The Antiparkinsonian Activity of CQA 206-291, a new D, Dopamine Receptor Agonist. Clin Neuropharmacol 1989;12:55-9.

\section{Neuromuscular paralysis in vipera aspis envenomation: pathogenetic} mechanisms

Vipera aspis is the most common agent of snake envenomation in Italy and Western Europe. ' Its bite affects coagulation and causes a shock syndrome with severe cardiovascular failure.

Neurotoxicity, clinically characterised by external ophthalmoplegia, is uncommon (two cases out of 205 patients bitten by vipera aspis) ${ }^{1}$ and difficult to explain because over neurotoxic substances have not been detected in vipera aspis venom. ${ }^{2}$ Our case suggests that the venom is neurotoxic.

A 20 year old herpetologist was bitten by a vipera aspis at the distal extremity of the index finger of the left hand. When he was admitted to the intensive care unit $(30 \mathrm{~min}$ utes later) he was unconscious (Glasgow Coma Scale 7), pale, tachycardic (170 beats/ min), tachypnoeic (50 breaths/min), without detectable peripheral pulses and blood pressure. There was a metabolic acidosis (pH 7.26) and disseminated intravascular coagulation. The left hand was oedematous. Centrifugal venous compression was applied on the left arm. Shock, metabolic failure and disseminated intravascular coagulation syndrome were treated with fresh frozen plasma, albumin, dextran, dopamine and adrenaline, $\mathrm{NaHCO}_{3}$ and heparin iv infusions. Cardiorespiratory function, metabolic balance and consciousness returned to normal within the following three hours.

Neurological examination revealed facial diplegia, pharyngolaryngeal paresis, bilateral ptosis and external ophthalmoplegia, with complete ocular immobility.

The strength of the trunk, limb and respiratory muscles, deep tendon reflexes, plantar and abdominal reflexes, and sensory functions were normal. Symptoms were not modified by iv administration of $10 \mathrm{mg}$ of edrophonium.

Neurophysiological studies of the facial nerves showed a low amplitude muscle action potential $(0.9 \mathrm{mv}-\mathrm{nv}>3 \mathrm{mv})$, with normal latency. Repetitive stimulation at low and high frequencies, tetanisation and stimulation with paired stimuli at stimulus intervals of less than $10 \mathrm{~ms}$ gave normal responses without signs of neuromuscular transmission defects. Blink reflex showed responses with normal latencies. Similar neurophysiological studies performed on other nerves (median, common peroneal and sural) were normal.

Five days from the onset of the disease the patient improved considerably and after 10 days, neurological examination and neurophysiological tests were normal. He was discharged after 10 days.

The lack of clinical involvement of motor, sensory and cerebellar pathways within the brainstem, together with the normal latency of blink reflex responses in this case, do not suggest an involvement of the brainstem possibly caused by oedema and/or dissemi- nated intravascular coagulation. ${ }^{3}$

The electrophysiological signs and the quick improvement of the clinical picture also lead us to exclude a neuropathic lesion and to hypothesise that a transient functional block of activation of a number of muscle fibres. This could be related to three possible mechanisms in particular: 1) a neuromuscular block; 2) a direct action on muscle fibres; 3) a block of depolarisation in the terminal portions of a number of motor nerve fibres.

A neuromuscular block may be related either to a presynaptic site of action of the venom, such as beta-bungarotoxin ${ }^{4}$ and antiacetylcholinesterase, or to a postsynaptic site of action, like alpha-bungarotoxin. ${ }^{2}$ None of these substances has been detected in vipera aspis and the electrophysiological findings of the reported case are neither consistent with a presynaptic nor a postsynaptic defect of neuromuscular transmission.

A direct myotoxic effect of animal toxin has been related to phospholipase $\mathrm{A} 2$ activity, which has been detected in all viperidae venoms so far investigated. ${ }^{2}$ Moreover some authors ${ }^{5}$ suggest that some toxins, like cardiotoxin of Dendroaspis jamesoni, can induce muscle fibre necrosis with a structural damage of the subneural apparatus. Nevertheless myonecrotic action is shown to be confined to the site of injection. ${ }^{67}$

The action of the toxin on the terminal portions of motor fibres could transiently block the conduction of a number of motor fibres by preventing their depolarisation. A lesion in this location is consistent with normal tests of neuromuscular transmission and with the rapid recovery of the amplitude of the muscle action potential as observed in our case. This mechanism has been hypothesised also in the neuromuscular paralysis induced by tick envenomation ${ }^{8}$ and by other biotoxins such as tetradotoxin.

Why the neurotoxic action of the vipera aspis venom appears to remain strictly localised in cephalic muscles remains unexplained. Peculiar physiological characteristics of cephalic motor units might be an explanation.

GIOVANNI ANTONINI
MAURIZIA RASURA
GIORGIO CONTI"
CONSALVO MATTIA"
Department of Neurological Science,
Institute of Anesthesiology and Resuscitation,"
University of Rome "La Sapienza",
Rome, Italy

Correspondence to: Dr Giovanni Antonini, Dipartimento di Scienze Neurologiche, III Clinica Neurologica, Viale dell'Università 30, 00185 Rome, Italy.

1 Pozio E. Venomous snake bites in Italy: epidemiological and clinical aspects. Trop Med Rarasit 1988;39:62-66.

2 Anthony T. Venoms: chemistry and molecular biology. New York: John Wiley, 1976.

3 Schwartzman RJ, Hill JB. Neurologic complications of disseminated intravascular coagulation. Neurology 1982;32:791-7.

4 Dreyer F, Penner R. The action of presynaptic snake toxins on membrane currents of mouse motor nerve terminals. J Physiol 1987; 386:455-63.

5 Duchen LW, Excell BJ, Patel R, Smith B. Changes in motor end-plates resulting from muscle fibre necrosis and regeneration: a light and electron microscope study of the effects of the depolarizing fraction (cardiotoxin) of of the depolarizing fraction (cardiotoxin) of 1974;21:391-417.

6 Harris JB, Karlsson E, Thesleff S. Effects of an isolated toxin from Australian Tiger Snake (Notechis Scutatus Scutatus) venom at the motechis Scutatus Scutatus) venom at the Pharmacol 1973;47:141-6.
7 Komory Y, Sugihara H. Biological study of muscle degenerating hemorrhagic factor from the venom of vipera aspis aspis (Aspic Viper). Int J Biochem 1988;20:1417-23.

8 Swift TR, Ignacio OJ. Tick paralysis. Electrophysiologic studies. Neurology 1975;25: $1130-33$.

9 Dettbarn WD, Higman HB, Rosemberg P, et al. Rapid and reversible block of electrical Rapid and reversible block of electrical activity by powerf

\section{MATTERS} ARISING

Comparison of two methods for measuring thermal thresholds

In their recent paper, ${ }^{1}$ Drs Levy, Abraham and Reid compare two techniques for measuring thermal thresholds in diabetic neuropathy. On the basis of their results they conclude that there is little to choose between the method of limits and the forced choice procedure of psychophysical analysis in the determination of thermal thresholds. We believe that their results and the conclusions based thereon are incorrect and a consequence of their experimental design.

When comparing two techniques attempting to measure the same parameter it is imperative that all variables are comparable and strictly controlled since they influence the accuracy of the final results. ${ }^{2-7} \mathrm{By}$ their own admission the authors have ignored a number of these variables as follows:

1) The reference skin temperatures for the Sensortek and Marstock methods are different $\left(30^{\circ} \mathrm{C}\right.$ and $32^{\circ} \mathrm{C}$ respectively). ${ }^{23}$ Neither is in the optimum range $34-35^{\circ} \mathrm{C}$ at which the variability of the thermal threshold measurements is minimal. ${ }^{67}$

2) The rate of temperature change in the Marstock technique is fixed. By comparison the rate of temperature change in the Sensortek technique, as described by the authors, varies not only during the application of a single stimulus but also for different applications. This is a source of variability. ${ }^{3467}$

3) In the Sensortek technique two stimuli of different modalities are applied to the skin more or less simultaneously; there is a tactile stimulus (when the thermode is applied to the skin) in addition to the specific thermal stimulus. It is particularly important that a pure thermal stimulus is applied without tactile cues as the latter has been shown to modify thermal sensation. 5

4) The duration of application of the thermode is poorly controlled in the Sensortek method. This will influence both the amount and rate of energy transferred to the receptor zone.

5) The pressure of application of the thermode to the skin is uncontrolled in both techniques. The authors state that the importance of this factor "in clinical testing has not been systematically investigated". This is incorrect. $^{67}$

6) The lack of calibration of heat transfer at the thermode-skin interface in both techniques does not allow for the variability of the thermal properties of the skin. ${ }^{89}$ 